\title{
Thermal Conductivity and Electrical Resistivity of Solid Iron at Earth's Core Conditions from First Principles
}

\author{
Junqing Xu, ${ }^{1}$ Peng Zhang, ${ }^{2}$ K. Haule, ${ }^{3}$ Jan Minar, ${ }^{4}$ Sebastian Wimmer, ${ }^{5}$ Hubert Ebert, ${ }^{5}$ and R. E. Cohen ${ }^{1,6, *}$ \\ ${ }^{1}$ Department of Earth and Environmental Sciences, LMU Munich, Theresienstrasse 41, 80333 Munich, Germany \\ ${ }^{2}$ School of Science, Xi'an Jiaotong University, Xi'an, Shaanxi, 710049, China \\ ${ }^{3}$ Department of Physics, Rutgers University, Piscataway, New Jersey 08854, USA \\ ${ }^{4}$ University of West Bohemia, New Technologies-Research Centre, Pilsen, Czech Republic \\ ${ }^{5}$ Department Chemie, Physikalische Chemie, University of Munich, D-81377 Munich, Germany \\ ${ }^{6}$ Extreme Materials Initiative, Geophysical Laboratory, Carnegie Institution for Science, Washington, D.C. 20015-1305, USA
}

(Received 26 March 2018; published 31 August 2018)

\begin{abstract}
We compute the thermal conductivity and electrical resistivity of solid hcp Fe to pressures and temperatures of Earth's core. We find significant contributions from electron-electron scattering, usually neglected at high temperatures in transition metals. Our calculations show a quasilinear relation between the electrical resistivity and temperature for hcp Fe at extreme high pressures. We obtain thermal and electrical conductivities that are consistent with experiments considering reasonable error. The predicted thermal conductivity is reduced from previous estimates that neglect electron-electron scattering. Our estimated thermal conductivity for the outer core is $77 \pm 10 \mathrm{~W} \mathrm{~m}^{-1} \mathrm{~K}^{-1}$ and is consistent with a geodynamo driven by thermal convection.
\end{abstract}

DOI: 10.1103/PhysRevLett.121.096601

The thermal conductivity of iron (Fe) and its alloys at Earth's core conditions is of central importance to understanding the thermal evolution of Earth's core and the energetics of the geomagnetic field [1-3]. A wide range of values for the thermal conductivity at core conditions has been predicted [3-8]. Previously, the thermal conductivity of iron at extreme conditions has been obtained from the electrical resistivity by applying the Wiedemann-Franz law: $\kappa=L T \sigma$, where $\kappa$ and $\sigma$ are the thermal and electrical conductivities, respectively, $\sigma$ is the inverse of electrical resistivity $\rho$, and $L$ is the conventional Lorenz number $L_{0}$ $\left(2.44 \times 10^{-8} \mathrm{~W} \Omega \mathrm{K}^{-2}\right)$ [9-11]. The Wiedemann-Franz law has generally not been verified for any material at extreme conditions. It can be derived under approximations [12] that would not apply under the high temperature of Earth's core. Direct measurements of thermal conductivity at conditions close to Earth's core conditions gave low values (e.g., $46 \mathrm{~W} \mathrm{~m}^{-1} \mathrm{~K}^{-1}$ ) [13] that would support the conventional thermal dynamo picture and are consistent with a geodynamo operating via thermal convection through Earth history. However, the thermal conductivity measurements and electrical resistivity measurements [11] are inconsistent, requiring extreme violations of the Wiedemann-Franz law. High values of thermal conductivity $\left(220 \mathrm{~W} \mathrm{~m}^{-1} \mathrm{~K}^{-1}\right)$ predicted by first-principles molecular dynamics (FPMD) with the Kubo-Greenwood formula within density functional theory (DFT) [6] are inconsistent with thermal convection of the core, requiring a different mechanism $[14,15]$. In addition to the relationship to heat transport, the electrical resistivity of iron and its alloys at Earth's core conditions is an important quantity for the geodynamo in itself, since a higher resistivity increases the dynamo dissipation.

We computed both the electron-phonon (e-ph) and electron-electron $(e-e)$ scattering contributions to electrical and thermal conductivity in solid hep iron. For each contribution, we have used two methods that have complementary approximations. First, we computed the $e$-ph contribution using the density functional perturbation theory (DFPT) and the inelastic Boltzmann transport equation [16] within ABINIT [17], see Supplemental Material [18]. Everywhere below, where we say "Boltzmann theory" we refer to electron-phonon scattering computed using the DFPT and Boltzmann transport theory. At high temperatures, the mean free path $l$ of electron due to $e$-ph scattering becomes comparable to the lattice constant so that resistivity saturation may become important [10]. The Boltzmann theory does not include saturation effects. We estimate such effects by applying the parallel resistor formula [32], whose reliability has been verified theoretically [33] and numerically [34,35]:

$$
\frac{1}{\rho_{e-\mathrm{ph}}}=\frac{1}{\rho_{\mathrm{sat}}}+\frac{1}{\rho_{B}},
$$

where $\rho_{B}$ is from the Boltzmann theory. $\rho_{\text {sat }}=\rho_{B} l_{B} / a$, where $a$ is the lattice constant and $l_{B}$ is the mean free path, i.e., the product of the relaxation time and Fermi velocity.

Second, we computed the $e$-ph contribution using the Korringa-Kohn-Rostoker (KKR) method with the coherent 


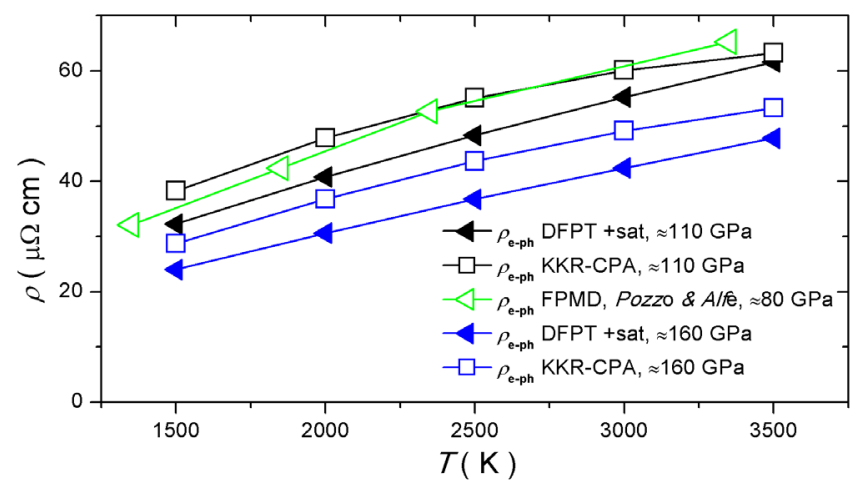

FIG. 1. Calculated resistivity of hep Fe at atomic volume 57.9 and 53.3 bohr $^{3}$, corresponding to about 110 and $160 \mathrm{GPa}$ at $2500 \mathrm{~K}[56,57] . \rho_{e-\mathrm{ph}}$ is the electron-phonon contribution of resistivity. DFPT represents calculating $\rho_{e \text {-ph }}$ using the DFPT + inelastic Boltzmann theory. "+ sat" includes resistivity saturation effects for the $e$-ph scattering using Eq. (1). "KKR-CPA" represents using the KKR-CPA method with the KuboGreenwood formula. FPMD is first-principles molecular dynamics with the Kubo-Greenwood formula. Both the KKR-CPA and the FPMD have naturally included resistivity saturation effects.

potential approximation (CPA) to model thermal lattice vibrations [36] with the SPRKKR code [37]. The electron scattering by phonons is computed from scattering by atomic displacements. Unlike the DFPT computations above, the KKR-CPA naturally includes resistivity saturation effects, which have been discussed as having important implications to transports properties in Earth's core [10]. We calculate the resistivity of hcp Fe at high temperatures using the KKR-CPA and the KuboGreenwood formula and find that the slope decreases with the temperature (Fig. 4), consistent with saturation effects. The theoretical results by the method above using the DFPT, Eq. (1), and the KKR-CPA are in good agreement with each other in a wide range of pressure and temperature (Fig. 1 and 4). However, the neglect of local environmental effects in the single-site KKR-CPA may lead to errors of transport properties.

We compared our thermal conductivity results with previous FPMD computations [5,6] and find that they agree well with those in Ref. [5], but less so with those in Ref. [6]. Within our methods, it seems to be easier to achieve good convergence than with FPMD. In practice, parameters in FPMD simulations, especially the cell size and number of $k$ points, are difficult to be converged for high-density metals. For instance, energy levels are discrete, and due to the finite supercell size in FPMD, the lowfrequency part of optical electrical and thermal conductivity and their dc values will depend on the choice of the width of the broadening function. We do not have such issues in our methods.

We computed the $e-e$ scattering contribution to electrical resistivity and thermal conductivity using the density functional theory + dynamical mean field theory (DFT+DMFT) $[38,39]$ with the continuous time quantum Monte Carlo impurity solver [40,41] and the KuboGreenwood formula with the EDMFTF code $[42,43]$. We use the method of Refs. [7,44], but correcting the factor of 2 error there due to the neglect of the two spin channels. The $e$-e scattering is additionally studied using the KKRDMFT [45] with the spin-polarized $T$ matrix + fluctuation exchange impurity solver $[18,46]$.

We add the separately computed $e$-ph and $e$-e contributions to give the total scattering rate; this is called Matthiessen's rule, which has broad experimental support. There are few studies considering corrections beyond Matthiessen's rule. Using the DFT+DMFT, $e$-ph coupling in FeSe was found to be enhanced due to electron correlations [47], and this has recently been verified experimentally [48]. For pure hcp Fe, electron correlations may also change the strength of $e$-ph coupling but probably not strongly. In recent work by Hausoel et al. [49], the authors report on DMFT calculations of molecular dynamics snapshots of fcc Ni and found that thermal disorder has only weak effects on electron correlations. Matthiessen's rule is expected to be broken when in the saturation region when the resistivity approaches the Ioffe-Regel limit, because there is essentially a minimum mean free paththe nearest-neighbor distance [50]. Previous studies [51,52] have shown that, for strongly correlated systems, resistivity can far exceed the Ioffe-Regel limit, corresponding to a very short mean free path. Therefore, when $e$-ph and $e$-e scattering contributions are comparable to each other, it may be suitable to consider saturation effects only on the $e$-ph part and apply Matthiessen's rule after having considered saturation. Since there is no evidence of the breakdown of Matthiessen's rule when considering $e-e$ and other scattering mechanisms, we assume the applicability of Matthiessen's rule.

Using this approximation, we compute the total thermal conductivity $\kappa_{\mathrm{tot}}=\left[\kappa_{e-\mathrm{ph}}^{-1}+\kappa_{e-e}^{-1}\right]^{-1}$, as in Ref. [53] for hydrogen plasma under extreme conditions. The ionic part of thermal conductivity is neglected, since it is much smaller than the electronic part in metals. As pointed out in Ref. [54], we observe relaxation time for the $e-e$ scattering $\tau_{e-e}$ being energy dependent, although we disagree with their claim of iron being a simple Fermi liquid at high temperatures [18]. Unlike $\rho_{e-e}$ being insensitive to the energy dependence of $\tau_{e-e}, \kappa_{e-e}$ can be considerably modified by its energy dependence. We find that the Lorenz number for the $e-e$ scattering, $L_{e-e}$, is reduced from the conventional one $L_{0}$ by $20 \%-45 \%$, or $1.4-2.0 \times 10^{-8} \mathrm{~W} \Omega \mathrm{K}^{-2}$, depending on the temperature and pressure. This leads to a Lorenz number for $\rho_{\text {tot }}$ and $\kappa_{\text {tot }}$ of $2.10-2.15 \times 10^{-8} \mathrm{~W} \Omega \mathrm{K}^{-2}$ at Earth's outer core conditions, from the core-mantle boundary $(\mathrm{CMB}, P=$ $136 \mathrm{GPa}$ and $T=4000 \mathrm{~K}$ ) to the inner core boundary (ICB, $P=330 \mathrm{GPa}$ and $T=6000 \mathrm{~K}$ ). 


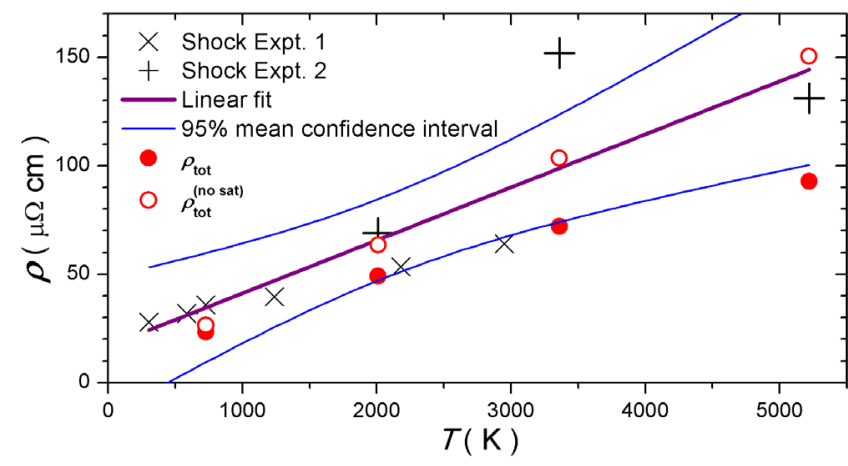

FIG. 2. Resistivity along the Hugoniot from shock data. $\times$ are from Ref. [9], and + are from Ref. [55]. $\rho_{\text {tot }}=\rho_{e-\mathrm{ph}}+\rho_{e-e}$, where $\rho_{e-\mathrm{ph}}$ considers resistivity saturation effects using Eq. (1), and $\rho_{e-e}$ is electrical resistivity due to the electron-electron scattering. $\rho_{\text {tot }}^{\text {(no sat) }}=\rho_{B}+\rho_{e-e}$, where $\rho_{B}$ is from Boltzmann theory and does not consider resistivity saturation. The purple line is the linear fit of the shock compression data. The blue lines are the 95\% mean confidence interval.

Our computed values of resistivity along the Hugoniot agree with the shock data from the experiments $[9,55]$ within the scatter (Fig. 2). We compared our computed isotropically averaged resistivity at conditions close to the $\mathrm{CMB}$ ones with diamond anvil cell (DAC) data [11] (Fig. 3). The computed resistivity is anisotropic, with $\rho_{a} / \rho_{c}=1.3$. We find that $\rho_{\text {sat }}$ is about $143 \mu \Omega \mathrm{cm}$ at $V=$ $47.8 \mathrm{bohr}^{3}$ /atom [18], a bit lower than the estimate by Gomi et al. [10]. Our resistivities are somewhat higher than the experimental data but broadly consistent, considering

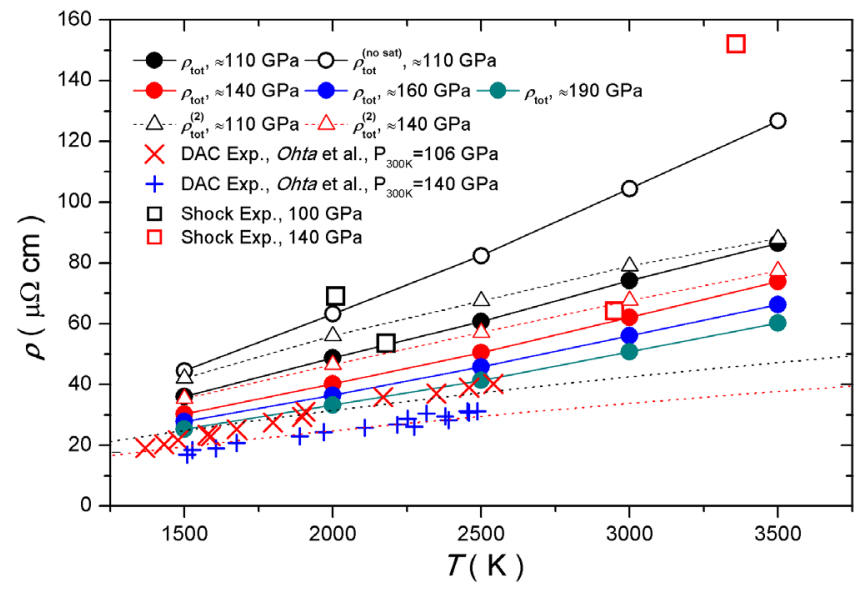

FIG. 3. Calculated resistivity at fixed atomic volumes-57.9, $54.9,53.3$, and $51.6 \mathrm{bohr}^{3}$, corresponding to pressures of about $110,140,160$, and $190 \mathrm{GPa}$ at $2500 \mathrm{~K}[56,57]$ — compared with shock data and experimental data by Ohta et al. [11]. $\rho_{\text {tot }}$ and $\rho_{\text {tot }}^{\text {(no sat) }}$ are total resistivity considering and not considering resistivity saturation effects, respectively. $\rho_{\text {tot }}^{(2)}$ is the total resistivity to which the $e$-ph contribution is calculated using the KKR-CPA and the Kubo-Greenwood formula. The dotted lines are their fits to the diamond anvil cell (DAC) data. the possibility of preferred orientation in the DAC experiments, temperature gradients, and the large size of the probe wires compared with the sample. Our calculations show a quasilinear relation between the total electrical resistivity and temperature for hcp Fe, against the relation used in the fit of their experimental data by Ohta et al., where the slope of resistivity decreases with the temperature. Including the $e$-e contribution, the absolute value and the slope of the total resistivity become larger, making the total resistivity more linear with the temperature. Applying such a quasilinear relation for extrapolation of experiments will increase their electrical resistivity at higher temperatures.

We compared our calculated resistivity with previous theoretical and experimental results at the inner core density of iron $\left(13.04 \mathrm{~g} \mathrm{~cm}^{-3}\right.$, atomic volume of 47.8 atomic units $=7.083 \AA^{3}$ ) (Fig. 4). Our $e$-ph results are slightly higher but in general agreement with the FPMD results [58]. Our total resistivity is in quite poor agreement with the extrapolation values of DAC data [11], consistent with their extrapolation not being accurate from overestimating saturation effects. In addition to possible experimental errors of temperatures in their measurements, the disagreement may be also due to their use of smaller $\rho_{\text {sat }}$ and the neglect of the $e$-e scattering in the temperature dependence of the resistivity in their extrapolation. At higher temperatures, the $e$-e scattering becomes more important and reaches about $35 \%$ of the $e$-ph value at Earth's core conditions.

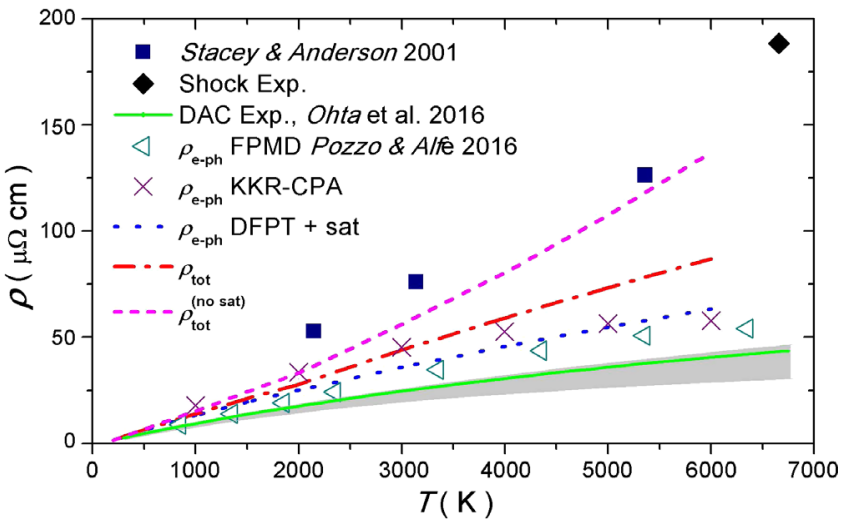

FIG. 4. Resistivity versus temperature of hcp Fe at Earth's inner core density. $\rho_{\text {tot }}$ and $\rho_{\text {tot }}^{\text {(no sat) }}$ are our calculated total resistivity considering and not considering resistivity saturation effects, respectively. The crosses are results using the KKR-CPA, which naturally includes saturation effects. The open triangle is FPMD results [58], where there is no $e-e$ contribution, at a little higher density. The navy squares are extrapolations to this density using the systematics of Stacey and Anderson [59] based on the melting curve (which has no fundamental justification). The dark diamond is an interpolation to this density and an extrapolation to the $6658 \mathrm{~K}$ temperature of previous shock compression results [9,55]. The green line is from Ref. [11] and is the extrapolation of their experimental data. 


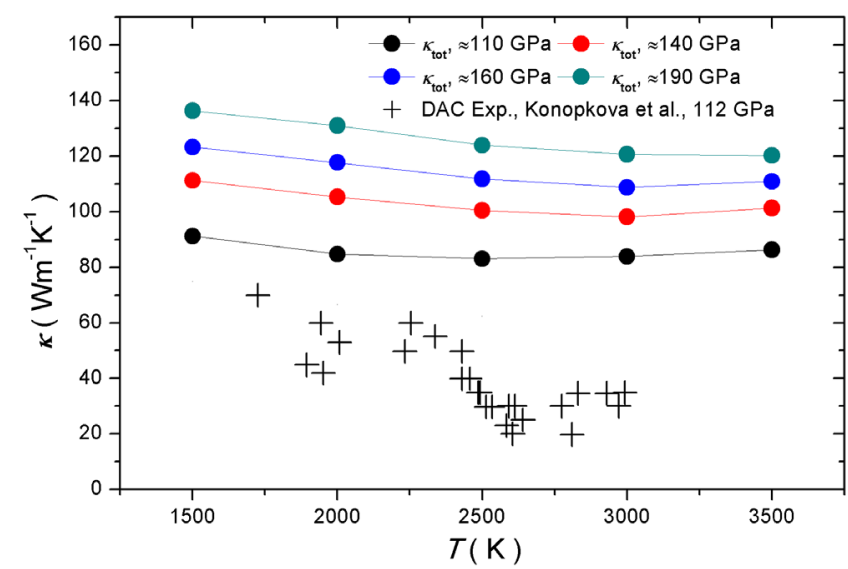

FIG. 5. Our calculated thermal conductivity of hcp Fe at fixed atomic volumes-57.9, 54.9, 53.3, and 51.6 bohr $^{3}$, corresponding to pressures about $110,140,160$, and $190 \mathrm{GPa}$ at $2500 \mathrm{~K}$ $[56,57]$ - compared with experimental data [13]. $\kappa_{\text {tot }}$ is the total thermal conductivity and is equal to $\kappa_{\mathrm{tot}}=\left[\kappa_{e-\mathrm{ph}}^{-1}+\kappa_{e-e}^{-1}\right]^{-1}$.

We compared our calculated thermal conductivity of hcp Fe at conditions close to $\mathrm{CMB}$ ones with experimental data [13] and find that the agreement is good at $2000 \mathrm{~K}$, but becomes poor above $2400 \mathrm{~K}$ (Fig. 5).

We calculate thermal conductivity at inner core density and obtain the theoretical electronic part of the thermal conductivity of pure solid iron, about $147 \mathrm{~W} \mathrm{~m}^{-1} \mathrm{~K}^{-1}$, at inner core conditions (Fig. 6). The agreement with the extrapolation model at $330 \mathrm{GPa}$ based on the experimental data at $112 \mathrm{GPa}$ [13] is quite poor. Except for possible errors in the measurements, and various assumptions made in our calculations, a possible reason for the disagreement may be that their extrapolation method is not accurate, since only the variation of thermal conductivity as a function of pressure and temperature due to $e$-ph scattering is considered.

Using previous estimates of thermal conductivity, i.e. with the theoretical thermal conductivity of liquid $\mathrm{Fe}-\mathrm{Si}$ or $\mathrm{Fe}-\mathrm{O}$ alloy (pure liquid $\mathrm{Fe}$ ) at $\mathrm{CMB}$ conditions, of about $100(140) \mathrm{W} \mathrm{m}^{-1} \mathrm{~K}^{-1}$, the heat loss from the core to the mantle by conduction is estimated to be 15 TW [6]. The total heat from the core is estimated to $8-16 \mathrm{TW}[61,62]$ so that the conventional thermal convection geodynamo model would probably fail. At CMB conditions, we find $\kappa \approx 97 \mathrm{~W} \mathrm{~m}^{-1} \mathrm{~K}^{-1}$ for pure solid hcp Fe (Fig. 5). Earth's outer core contains light elements of the order of $20 \%$, and light elements will probably decrease the $e$-ph scattering contribution to thermal conductivity by $10 \%-30 \%[5,6,10]$. In addition, melting will decrease the density and may further lead to an $\approx 10 \%$ reduction of both the $e$-ph contribution [60] and the $e-e$ contribution. The thermal conductivity would accordingly be about $77 \mathrm{~W} \mathrm{~m}^{-1} \mathrm{~K}^{-1}$. The corresponding heat conduction down the core adiabat will be about 9-12 TW, depending on the choice of core parameters, e.g., specific heat capacity, CMB temperature, etc., $[2,3,6]$.

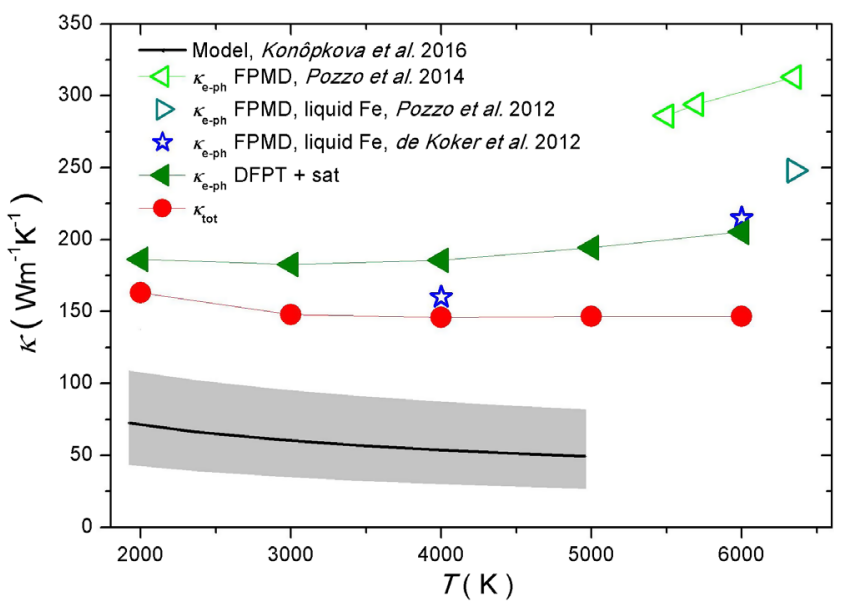

FIG. 6. Calculated thermal conductivity of solid hep Fe at Earth's core density, $13.04 \mathrm{~g} \mathrm{~cm}^{-3}$, or atomic volume $47.8 \mathrm{bohr}^{3}$. At $6000 \mathrm{~K}$, the corresponding pressure is about $305 \mathrm{GPa} . \kappa_{\mathrm{tot}}$ is our calculated total thermal conductivity using the DFPT and DMFT. The black line is the extrapolation model at $330 \mathrm{GPa}$ based on the experimental data at $112 \mathrm{GPa}$ [13]. A uncertainty envelope of the model (gray) is given according to our estimate that their experimental data at $2000 \mathrm{~K}$ and the extrapolation to pressure $330 \mathrm{GPa}$ may both have errors of $10 \%-30 \%$ and the thermal conductivity may decrease slower than $T^{-0.5}$, as assumed in their model. The green open triangles are the theoretical values of thermal conductivity at $329 \mathrm{GPa}$ due to only $e$-ph scattering using FPMD [60], which includes saturation effects consistent with system size and $k$-point sampling. The dark cyan open triangle and blue open stars are the theoretical thermal conductivity of liquid Fe due to only $e$-ph scattering using FPMD $[5,6]$.

Another candidate phase of solid Fe alloy at Earth's core conditions is bcc [63,64], which is dynamically unstable, so we cannot fully apply DFPT to compute transport properties; for completeness we estimate the $e$-ph scattering contribution of bcc Fe neglecting the unstable modes and calculate the $e-e$ scattering contribution using DFT+DMFT. We find that the total resistivity of bcc Fe is different from that of hcp Fe by several percent. All of the above results are for solid iron, but experiments on many materials show that melting typically increases resistivity by $5 \%-10 \%$. We computed the effects of melting on iron by applying DFT+DMFT to snapshots of liquid Fe from firstprinciples molecular dynamics [18] and find that scattering rates due to $e$ - $e$ scattering around the chemical potential are about $20 \%-40 \%$ larger than those of solid $\mathrm{Fe}$ at similar conditions, and that the thermal conductivity of liquid iron at Earth's core conditions is reduced by about 10\% from solid iron. A full discussion of results for liquid iron and its alloys will be discussed in another work, as it is a separate ongoing study.

Our final estimates for the thermal conductivity for pure solid hcp iron at Earth's inner core conditions is $\kappa=147$ and $97 \mathrm{~W} \mathrm{~m} \mathrm{~K}$ at the core-mantle boundary temperature and pressure, and $77 \pm 10$ for liquid iron alloy in the outer 
core. This is consistent with a thermally convection driven dynamo throughout Earth history, which requires $\kappa=100 \mathrm{~W} \mathrm{~m} \mathrm{~K}$ for a CMB heat flow of about $15 \mathrm{TW}$ and temperature $T_{\mathrm{CMB}}=4000 \mathrm{~K}[65]$.

We thank Peter Driscoll, Jung-Fu Lin, and Youjun Zhang for helpful discussions. This work was supported by the European Research Council Advanced Grant ToMCaT and by the Carnegie Institution for Science. The authors gratefully acknowledge the Gauss Centre for Supercomputing (GCS) e.V. for funding this project by providing computing time on the GCS Supercomputer SuperMUC at Leibniz Supercomputing Centre (LRZ). P.Z. acknowledges support of National Science Foundation of China (Grant No. 11604255). J. M. further thanks for the support from the Computational and Experimental Design of Advanced Materials with New Functionalities (CEDAMNF) project (CZ.02.1.01/0.0/0.0/ 15_003/0000358) of Czech ministerium MSMT.

*rcohen@carnegiescience.edu

[1] S. Labrosse, Phys. Earth Planet. Inter. 140, 127 (2003).

[2] F. Nimmo, Treatise on Geophysics: Energetics of the Core (Elsevier, New York, 2015).

[3] F. Stacey and D. Loper, Phys. Earth Planet. Inter. 161, 13 (2007).

[4] X. Sha and R. E. Cohen, J. Phys. Condens. Matter 23, 075401 (2011).

[5] N. de Koker, G. Steinle-Neumann, and V. Vlček, Proc. Natl. Acad. Sci. U.S.A. 109, 4070 (2012).

[6] M. Pozzo, C. Davies, D. Gubbins, and D. Alfe, Nature (London) 485, 355 (2012).

[7] P. Zhang, R. Cohen, and K. Haule, Nature (London) 517, 605 (2015).

[8] R. A. Secco, Phys. Earth Planet. Inter. 265, 23 (2017).

[9] R. Keeler and A. Mitchell, Solid State Commun. 7, 271 (1969).

[10] H. Gomi, K. Ohta, K. Hirose, S. Labrosse, R. Caracas, M. J. Verstraete, and J. W. Hernlund, Phys. Earth Planet. Inter. 224, 88 (2013).

[11] K. Ohta, Y. Kuwayama, K. Hirose, K. Shimizu, and Y. Ohishi, Nature (London) 534, 95 (2016).

[12] M. Jonson and G. D. Mahan, Phys. Rev. B 21, 4223 (1980).

[13] Z. Konôpková, R. S. McWilliams, N. Gómez-Pérez, and A. F. Goncharov, Nature (London) 534, 99 (2016).

[14] J. G. O'rourke and D. J. Stevenson, Nature (London) 529, 387 (2016).

[15] J. Badro, J. Siebert, and F. Nimmo, Nature (London) 536, 326 (2016).

[16] P. Allen, Phys. Rev. B 17, 3725 (1978).

[17] X. Gonze et al., Comput. Phys. Commun. 205, 106 (2016).

[18] See Supplemental Material at http://link.aps.org/ supplemental/10.1103/PhysRevLett.121.096601 for more technical details and theoretical results, which additionally includes Refs. [19-31].

[19] S. Goedecker, M. Teter, and J. Hutter, Phys. Rev. B 54, 1703 (1996).
[20] J. P. Perdew, K. Burke, and M. Ernzerhof, Phys. Rev. Lett. 77, 3865 (1996).

[21] G. K. Madsen and D. J. Singh, Computer Physics Communications 175, 67 (2006).

[22] K. Schwarz, P. Blaha, and G. K. Madsen, Computer Physics Communications 147, 71 (2002).

[23] J. Minár, J. Phys. Condens. Matter 23, 253201 (2011).

[24] J. Schött, I. L. M. Locht, E. Lundin, O. Grånäs, O. Eriksson, and I. Di Marco, Phys. Rev. B 93, 075104 (2016).

[25] P. Nozières and D. Pines, Theory of Quantum Liquids (Westview, Boulder, CO, 1999).

[26] C. Herring, Phys. Rev. Lett. 19, 167 (1967).

[27] C. Herring, Phys. Rev. Lett. 19, 684 (1967).

[28] A. V. Chubukov and D. L. Maslov, Phys. Rev. B 86, 155136 (2012).

[29] H. M. Milchberg, R. R. Freeman, S. C. Davey, and R. M. More, Phys. Rev. Lett. 61, 2364 (1988).

[30] J. Abraham and B. Deviot, J. Less-Common Met. 29, 311 (1972).

[31] P. Giannozzi et al., J. Phys. Condens. Matter 21, 395502 (2009).

[32] H. Wiesmann, M. Gurvitch, H. Lutz, A. Ghosh, B. Schwarz, M. Strongin, P. Allen, and J. Halley, Phys. Rev. Lett. 38, 782 (1977).

[33] P. B. Allen and B. Chakraborty, Phys. Rev. B 23, 4815 (1981).

[34] W. Schiller and G. Langowski, J. Phys. F 12, 449 (1982).

[35] Y. Werman, S. A. Kivelson, and E. Berg, npj Quantum Mater. 2, 7 (2017).

[36] H. Ebert, S. Mankovsky, K. Chadova, S. Polesya, J. Minar, and D. Kodderitzsch, Phys. Rev. B 91, 165132 (2015).

[37] H. Ebert, D. Koedderitzsch, and J. Minar, Rep. Prog. Phys. 74, 096501 (2011).

[38] A. Georges, G. Kotliar, W. Krauth, and M. J. Rozenberg, Rev. Mod. Phys. 68, 13 (1996).

[39] G. Kotliar, S. Y. Savrasov, K. Haule, V. S. Oudovenko, O. Parcollet, and C. Marianetti, Rev. Mod. Phys. 78, 865 (2006).

[40] K. Haule, Phys. Rev. B 75, 155113 (2007).

[41] E. Gull, A. J. Millis, A. I. Lichtenstein, A. N. Rubtsov, M. Troyer, and P. Werner, Rev. Mod. Phys. 83, 349 (2011).

[42] K. Haule, C.-H. Yee, and K. Kim, Phys. Rev. B 81, 195107 (2010).

[43] K. Haule and T. Birol, Phys. Rev. Lett. 115, 256402 (2015).

[44] P. Zhang, R. Cohen, and K. Haule, Nature (London) 536, 112 (2016).

[45] J. Minár, L. Chioncel, A. Perlov, H. Ebert, M. I. Katsnelson, and A. I. Lichtenstein, Phys. Rev. B 72, 045125 (2005).

[46] L. V. Pourovskii, M. I. Katsnelson, and A. I. Lichtenstein, Phys. Rev. B 72, 115106 (2005).

[47] S. Mandal, R. E. Cohen, and K. Haule, Phys. Rev. B 89, 220502 (2014).

[48] S. Gerber et al., Science 357, 71 (2017).

[49] A. Hausoel, M. Karolak, E. Şaşıoğlu, A. Lichtenstein, K. Held, A. Katanin, A. Toschi, and G. Sangiovanni, Nat. Commun. 8, 16062 (2017).

[50] M. Gurvitch, Phys. Rev. B 24, 7404 (1981).

[51] O. Gunnarsson, M. Calandra, and J. Han, Rev. Mod. Phys. 75, 1085 (2003). 
[52] F. Rizzo, E. Cappelluti, and L. Pietronero, Phys. Rev. B 72, 155113 (2005).

[53] M. P. Desjarlais, C. R. Scullard, L. X. Benedict, H. D. Whitley, and R. Redmer, Phys. Rev. E 95, 033203 (2017).

[54] L. Pourovskii, J. Mravlje, A. Georges, S. Simak, and I. Abrikosov, New J. Phys. 19, 073022 (2017).

[55] Y. Bi, H. Tan, and F. Jing, J. Phys. Condens. Matter 14, 10849 (2002).

[56] A. Dewaele, P. Loubeyre, F. Occelli, M. Mezouar, P. I. Dorogokupets, and M. Torrent, Phys. Rev. Lett. 97, 215504 (2006).

[57] X. Sha and R. E. Cohen, Phys. Rev. B 81, 094105 (2010).

[58] M. Pozzo and D. Alfè, SpringerPlus 5, 256 (2016).
[59] F. D. Stacey and O. L. Anderson, Phys. Earth Planet. Inter. 124, 153 (2001).

[60] M. Pozzo, C. Davies, D. Gubbins, and D. Alfè, Earth Planet. Sci. Lett. 393, 159 (2014).

[61] T. Lay, J. Hernlund, and B. A. Buffett, Nat. Geosci. 1, 25 (2008).

[62] B. Wu, P. Driscoll, and P. Olson, J. Geophys. Res.: Solid Earth 116, B12112 (2011).

[63] A. B. Belonoshko, T. Lukinov, J. Fu, J. Zhao, S. Davis, and S. I. Simak, Nat. Geosci. 10, 312 (2017).

[64] L. Vocadlo, I. G. Wood, M. J. Gillan, J. Brodholt, D. P. Dobson, G. D. Price, and D. Alfe, Phys. Earth Planet. Inter. 170, 52 (2008).

[65] P. Driscoll and D. Bercovici, Phys. Earth Planet. Inter. 236, 36 (2014). 